\title{
Psychosis following temporal lobe surgery: a report of six cases
}

\author{
C J Mace, M R Trimble
}

\begin{abstract}
Six consecutive patients who had had temporal lobe surgery for epilepsy, and been referred for psychiatric assessment of psychotic symptoms, are reported. Their symptoms (a delusional depression, four schizophrenia-like illnesses, and a case of Capgras' syndrome) are discussed in relation to the possible role of their operations, all of which were on the right hemisphere.
\end{abstract}

Temporal lobe surgery, notably the anterior lobectomy pioneered by both Bailey and Falconer $^{12}$ is well established as a valuable treatment for chronic but localised epilepsy, especially when other forms of treatment have failed. During its development, considerable attention has always been paid to neurological ${ }^{3}$ and cognitive ${ }^{4}$ deficits that may arise for the first time following operation, with important amendments of operative technique resulting. Subsequently, attention paid to the outcome of surgery in terms of patients' social functioning had a useful impact on rehabilitation. ${ }^{5}$ The occurrence, however, of frank psychiatric illness, both before and after surgery, has been relatively neglected. ${ }^{6}$

This report summarises the only six cases of post-operative psychotic illness among patients having previous temporal lobe surgery for epilepsy that were referred for psychiatric assessment at a specialist centre over a 10 year period. The original operations had been performed at the National Hospitals in London in four of the cases, at the Maudsley Hospital in one, and at the University of California at Los Angeles for the remaining case. Common features noted in their surgical and psychiatric histories will be discussed in an attempt to highlight the need for greater understanding of relationships between psychiatric morbidity and surgical intervention.

\section{Institute of Neurology, London \\ C J Mace \\ National Hospital for Nervous Diseases, London M R Trimble \\ Correspondence to: Dr Mace, Institute of Neurology, Queen Square, London WCIN 3BG, UK \\ Received 3 May 1990 and in final revised form \\ 5 December 1990.
Accepted 10 December 1990} A epilepsy at the age of 13 referred at the age of 20 while in a rehabilitation unit. Five months previously he had a craniotomy with extirpation of a large right sided epidermoid cyst immediately medial to the temporal lobe. His epilepsy had been completely relieved following operation; a delusional depression was diagnosed.

There was no history of psychiatric illness or epilepsy in this patient's family, although his family circumstances and emotional development had been disturbed before the onset of seizures. He had been academically unsuccessful at school, having had a history of aggressive behaviour there and afterwards, and had spent less than a year in employment on leaving school. Epilepsy began at the age of 13, two weeks subsequent to a minor head injury, in the form of both partial and generalised seizures. Right focal EEG abnormalities were present, and by the age of 19, when fits were more frequent, left sided peripheral motor signs were observed. A CT scan confirmed the presence of a space occupying lesion, which was removed. With the exception of an episode of status immediately postoperatively, and a seizure associated with change of medication a year later, he had remained fit-free. Following the operation however, he was reported to be lacking in confidence, and three months later to have become very preoccupied with his sexual potency, although he remained physiologically competent in this respect. He became increasingly withdrawn before reviews by two psychiatrists confirmed he was severely depressed and suicidal five months postoperatively. He made a slow recovery over several months from this state following combined treatment with mianserin and haloperidol. At the height of his depression he expressed nihilistic beliefs that he had lost everything and could no longer feel anything, before taking a substantial overdose of anticonvulsants. He remained preoccupied with the idea that irrevocable damage had been done to his sexual functioning since his operation, and this was linked to a set of beliefs concerning disfigurements to his anatomy, and how parts of his body controlled one another in ways having no basis in medical fact. He refused ever to fully discuss these, making it uncertain, after the eventual remission of his depression, whether they had fully disappeared. Over three years of subsequent follow up there had been no further psychotic episodes, he remained free of seizures and auras, obtained a driving licence, and was able to move to independent accommodation.

\section{Case B}

Case B was a 21 year old right handed shop worker whose epileptic history began at the age of two, and who was attending a neurology outpatient clinic at the time of referral. He had a right temporal lobectomy at 
the age of nineteen that had removed an area of mesial temporal sclerosis and completely relieved his epilepsy. A schizophreniform psychosis was diagnosed.

His early upbringing was disturbed and complicated: lack of contact with his natural parents has rendered details of his family history uncertain, although it is likely one parent had suffered from serious mental illness. After febrile convulsions at the age of two, the patient had absence attacks from the age of five, complex partial seizures associated with automatism from the age of seven, and generalised seizures from late childhood, the latter two proving refractory to treatment. Despite these handicaps, he had been able to leave school with some CSE certificates, appears to have been a shy and sensitive personality, but to have had no previous psychiatric history apart from some counselling at the age of fourteen.

After proceeding to surgery, hippocampal sclerosis was discovered in the removed right temporal lobe, and he was subsequently completely free of seizures. No change in personality was evident, and he returned to his former job and accommodation. Referral for psychiatric assessment came after conversational remarks betrayed a delusional preoccupation that had been present from two years after the operation. His mental state revealed a tightly encapsulated set of delusions concerning operations he said were being performed daily on his memory and thinking processes. This occurred by remote action, without his consent, and for which he held a new neighbour responsible. There was also clinical evidence of intermittent auditory hallucinosis and thought echo: administration of the Present State Examination ${ }^{7}$ returned a diagnosis of nuclear schizophrenia. In other respects his behaviour was socially appropriate, and his affect was judged to be unimpaired. Psychometric examination provided no evidence of cognitive impairment since the time of his operation. His psychotic symptoms responded well to phenothiazine medication, although the patient developed little insight into the nature of his symptoms. Although he reported occasional aura-like experience in the first post-surgical year, he remained seizure free and returned to his former employment.

\section{Case $C$}

Case $\mathrm{C}$ was a right handed man who had had epilepsy since the age of seven, been in institutional care since childhood, and who was referred at the age of 28 . He had a right temporal lobectomy five years earlier with removal of an oligodendroglioma, but experienced only temporary respite from his epilepsy. A schizophreniform psychosis was diagnosed.

There had been no known family history of epilepsy or psychiatric disorder. A parental divorce had already complicated his emotional development before onset of epilepsy, which dated from a severe bout of measles at the age of seven. He attended special boarding schools from that time, while his epileptic fits gradually altered, as absence attacks gave way to serial tonic-clonic seizures, and increased in frequency. Throughout, he was noted to be episodically aggressive, often immediately following an ictus. Brief experiences of a stereotyped and indistinct auditory hallucination, noted at the age of 22 , had been attributed to a seizure phenomenon. Anticonvulsant therapy was increasingly ineffective, and operation followed discovery of a lesion on CT scan.

The right temporal lobectomy included excision of a mass which transpired to be a oligodendroglioma, and post operative radiotherapy was given. The patient had a residual left hemiplegia that remitted slowly but incompletely over the next six years, and his epilepsy was initially considerably improved. Five years after the operation, however, he was again subject to daily generalised seizures. A worsening in behaviour was also noted at this time: he had become more irritable, was refusing to manage his own affairs as he had formerly, and made dramatic, highly public suicidal threats. Psychiatric examination revealed subjective dysphoria along with formal thought disorder, a delusory belief that passive control of his actions was being exerted by a co-resident of his institution, and an auditory hallucinosis congruent with this that had reportedly been present for a year. The patient betrayed no insight into these symptoms which appeared to remit in response to regular injection of fluphenazine decanoate, although his epilepsy remained very difficult to control, and he remained incapable of work or independent living. Psychometric testing confirmed that his intellectual functioning remained poor, and that memory in particular had deteriorated considerably since the time of surgery. A CT scan at the time of referral confirmed that no recurrence of his tumour was evident.

\section{Case D}

Case D was a hospitalised left handed 43 year old man who had developed epilepsy at the age of ten, and who had first had a schizophrenic illness at the age of 24 . He received a right temporal lobectomy at the age of 23 that had not removed any histologically abnormal tissue, and that had little impact on his seizures. Schizophrenia was again diagnosed.

There had been no family history of epilepsy; a paternal aunt had been admitted to hospital for a hallucinatory illness in her thirties, dying shortly afterwards. The patient's development was apparently unremarkable before onset of epilepsy at the age of ten, and he continued to perform well academically, passing general certificate of education subjects. He then attempted a series of skilled jobs but has had no regular employment since the age of nineteen. From the age of ten onwards, his epilepsy progressed from absence attacks, to a variety of complex partial seizures to include generalised seizures and bouts of status epilepticus. As his epilepsy had worsened, the patient became dysphasic post-ictally, and after a hospital admission for status epilepticus at the age of twenty three he had been transferred to a 
psychiatric unit for observation. An organic brain syndrome characterised by clouding of consciousness and post-ictal dysphasia was diagnosed, with no evidence of schizophrenia being discovered.

A right sided temporal lobectomy was performed at the age of 23. Although pre-operative amytal injection had indicated that speech was represented in the left hemisphere, bilateral representation was assumed when stimulation of the right superior temporal gyrus produced speech arrest during operation. The right superior temporal gyrus was therefore spared, but with medial excision including the hippocampus. Post-operatively he developed a left spastic hemiparesis, relieved by removal of a haematoma; he continued to suffer right-sided deafness and a left hemianopia. In addition, relatives had reported a personality change immediately after surgery, with the patient becoming aloof and distant. It is likely he expressed ideas of reference periodically from five months post-operatively. A psychiatrist's assessment at the age of 24 noted the presence of thought disorder, primary delusions, passivity phenomena and affective changes. He was admitted to hospital at the age of 25 following an overdose when, in addition to affective symptoms, delusions of persecution, thought broadcasting and passivity phenomena were recorded. Impairment of the stream of speech was then attributed to schizophrenic thought disorder, which improved along with the other features in response to phenothiazine medication. He had continued to require treatment up until referral to this centre, and to have been institutionally dependent. His affect was flat, there was marked thought disorder, and in addition to thought broadcasting he expressed passivity feelings concerning electrical influences on his brain, and uncertainty about his gender. Follow up over a further two year period showed that episodes of marked affective and psychotic symptomatology had come to alternate with periods of relatively poor epileptic control.

\section{Case E}

Case $\mathrm{E}$ was an ambidextrous single man receiving an inpatient psychiatric assessment at the age of 34. He had developed epilepsy at the age of one, and had first been admitted to hospital for a schizophreniform episode at the age of 31 , having had a right temporal lobectomy at the age of 22 . This had removed an area of mesial temporal sclerosis, and had moderated but not cured his seizures. A schizophrenic episode was diagnosed.

There had been no family history either of epilepsy or mental illness. His family background was disturbed, and he had known only one of his natural parents. His epilepsy had been continuous from one year of age, onset being three months after a right-sided head injury, and taking the form initially of complex partial seizures, with a minority of these subsequently leading to generalised attacks. Throughout childhood the patient had been hyperactive and prone to aggressive and highly antisocial behaviour, attending a number of different special schools. Academic achievements were very modest; he is reported to have become more withdrawn in personality from the age of eleven. The lobectomy was performed after a worsening response to anticonvulsant treatment, and a two year period free of seizures followed, after which he had continued to have both forms of attacks albeit less frequently than in adolescence.

The patient had been admitted briefly to a psychiatric hospital at the age of nineteen, following an overdose during a family row; some emotional lability was noted but no schizophrenic symptoms were recorded or specific treatment given. He had a further psychiatric admission one year after operation, apparently purely to relieve his family, and again without treatment. He continued to have spontaneously resolving depressive episodes of two to three weeks duration over ensuing years while remaining incapable of work or living independently. Schizophrenic symptoms were recorded for the first time during his next psychiatric admission at the age of 32 , in the form of auditory hallucinations in the third person, ideas of reference, a belief that he was being controlled by a television set, and reports of thought withdrawal. These then resolved over two months following rationalisation of his anticonvulsant regime. There were further brief recurrences of these symptoms before a more prolonged episode at the age of 34, which accompanied a depressive mood change. In addition to ideas of reference and persecutory delusions, he reported thought withdrawal and a belief that a third party was controlling his actions. He also expressed recurrent doubts concerning his own gender. On this occasion neuroleptic medication was instituted, with the apparent relief of all symptoms.

\section{Case F}

Case $\mathrm{F}$ was a right handed married man originally assessed as an outpatient at the age of 41 with a five year history of rage attacks, who had had complex partial seizures from the age of 19 . A diagnosis of ictal automatism was made at that time. He had a right sided temporal abscess drained surgically when 19 , and went on to have a right temporal lobectomy that completely relieved his epilepsy at the age of 43. Within a further month he developed a psychotic illness in the form of an atypical delusional state accompanied by marked depressive symptomatology.

There was no family history of epilepsy or psychiatric disorder. He had been repeatedly singled out for punishment by his parents, and had had an unhappy and interrupted school career. He received only a basic education and was working at the time of developing the temporal abscess secondary to a middle ear infection. The complex partial seizures started two months later, despite prophylactic medication. Generalisation of some seizures developed at the same time as the rage attacks, for which he could be amnesic. Family tensions had been evident for some years before this. His seizures proved increasingly resistant to medication and, apparently secondary to this, 
the patient had admitted to depressive symptoms and suicidal thoughts before surgery. Following his lobectomy at 43 years he had a dense left spastic paraparesis accompanied by left hemianesthesia, and infarction in the posterior part of the right hemisphere was also noted.

Within a month of surgery he was subject to intense feelings that his surroundings were not real, and spontaneously voiced abnormal beliefs that were both nihilistic (for example, that people around him were actually dead), and the so-called "delusion of doubles" (that is, stating that people around him had been replaced by impostors). He had two psychiatric admissions in quick succession with these complaints, being put on neuroleptic medication with benefit during the first, with the readmission following an isolated seizure and failure to comply with medication. Over a two year follow up period he was apparently seizure free, but was subject to brief episodes of irritability or simple visual hallucinations such as seeing lines in front of him. He also complained of impotence. With much investigation it transpired that although his cerebrovascular state had compromised his erectile function, marital problems contributed significantly to this difficulty.

\section{Discussion}

These six patients with epilepsy all developed psychotic illnesses for the first time following temporal lobe surgery. There was little consistency in the timing of the appearance of symptoms, which had ranged from the first post-surgical month (case $F$ ) to the ninth postsurgical year (case D), (see table).

Nevertheless, it was evident that affective symptoms were most evident in those appearing earliest (cases $\mathrm{F}$ and $\mathrm{A}$ ). These were also two cases that did not comprise typical schizophrenic syndromes, severe depressive symptomatology dominating the picture in case $A$, while case $F$, in addition to a depressed affect, exhibited the "delusion of doubles" pathognomonic of Capgras' syndrome ${ }^{9}$ in his belief that members of his family had been

Table Comparative features of the six cases

\begin{tabular}{|c|c|c|c|c|c|c|}
\hline Patient & $A$ & $B$ & $C$ & $D$ & $E$ & $F$ \\
\hline $\begin{array}{l}\text { Sex: } \\
\text { Handedness: } \\
\text { Family psychiatric history: } \\
\text { Age of epilepsy onset: } \\
\text { Age at operation: } \\
\text { Side of operation: } \\
\text { Lobectomy? } \\
\text { Age of onset of psychosis: }\end{array}$ & $\begin{array}{l}M \\
\mathrm{R} \\
-13 \\
20 \\
\mathrm{R} \\
\overline{20}\end{array}$ & $\begin{array}{l}M \\
R \\
+ \\
2 \\
19 \\
R \\
+ \\
21\end{array}$ & $\begin{array}{l}\mathrm{M} \\
\mathrm{R} \\
- \\
7 \\
23 \\
\mathrm{R} \\
+ \\
28\end{array}$ & $\begin{array}{l}M \\
L \\
+ \\
10 \\
23 \\
R \\
+ \\
24\end{array}$ & $\begin{array}{l}\mathrm{M} \\
\mathrm{R} \\
- \\
1 \\
22 \\
\mathrm{R} \\
+ \\
32\end{array}$ & $\begin{array}{l}M \\
R \\
-19 \\
43 \\
R \\
\overline{43}\end{array}$ \\
\hline $\begin{array}{l}\text { Post operative symptoms: } \\
\text { depression? } \\
\text { passivity phenomena? } \\
\text { other delusions? } \\
\text { auditory hallucinations? } \\
\text { thought disorder? }\end{array}$ & $\begin{array}{l}++ \\
+ \\
+ \\
-\end{array}$ & $\begin{array}{l}- \\
+ \\
\overline{+} \\
-\end{array}$ & $\begin{array}{l}+ \\
+ \\
+ \\
+\end{array}$ & $\begin{array}{l}+ \\
+ \\
+ \\
+\end{array}$ & $\begin{array}{l}+ \\
+ \\
+ \\
+\end{array}$ & $\begin{array}{l}++ \\
+ \\
+ \\
-\end{array}$ \\
\hline $\begin{array}{l}\text { Follow up data: } \\
\text { length (years): } \\
\text { control of epilepsy post-op: } \\
\left.\text { [Ratings according to Engel }(1987)^{8}\right]\end{array}$ & ID & $\begin{array}{r}2 \\
\text { IB }\end{array}$ & $\stackrel{5}{\text { IVB }}$ & $\begin{array}{l}20 \\
\text { IVA }\end{array}$ & $\begin{array}{l}12 \\
\text { IIIA }\end{array}$ & $\underset{\text { IB }}{2}$ \\
\hline $\begin{array}{l}\text { Social adjustment post-op: } \\
\text { (pre-op): }\end{array}$ & $\stackrel{\star \star \star \star}{(\star)}$ & $\begin{array}{c}\star \star \star \\
(\star \star\end{array}$ & $\stackrel{\star}{(\star)}$ & $\stackrel{\star \star}{(\star \star})$ & $\stackrel{\star}{\star \star}$ & $\stackrel{\star \star \star}{(\star \star})$ \\
\hline
\end{tabular}

replaced by impostors who bore a close physical resemblance to them.

Among the four patients that unequivocally demonstrated schizophrenic symptomatology, lobectomy has allowed pathological comparisons. A diverse range of neuropathology was uncovered, shedding no new light on relationships between symptomatology and lesion type. However, the outcome with respect to both social adjustment and seizure frequency was found to parallel the observations of Bruton, ${ }^{10}$ being poor for the patients having an oligodendroglioma (case C) and no pathology in the resected lobe (case D), and relatively good for the patients with mesial temporal sclerosis (cases B and E). There was no consistent relationship between the subsequent control of seizures and the appearance of psychotic symptoms across this series. It was noted, however, that all patients had had secondary generalised seizures in addition to purely focal attacks.

When comparing the patients' psychiatric symptomatology, an interesting consistency emerges among these same four patients (cases B-E). While some affective symptoms accompanied the schizophrenic features, the authors were struck by the prominence of passivity phenomena in each case. In these, a disturbance of volition leads to the experience that particular thoughts, feelings or actions are entirely subject to the control of an external force or agency. When passivity phenomena occurred, they were the subject of delusional interpretations, but elaborate belief systems had not been erected around them. Hallucinatory experience, when present, was not the dominant feature of the clinical picture in any of the cases. In previous literature concerning the phenomenology of epileptic psychosis, passivity phenomena have either been construed as an aspect of chronic delusional symptoms, ${ }^{11}$ or as part of a set of diagnostically equivalent "nuclear" schizophrenic symptoms which include auditory hallucinosis, as promoted by advocates of the Present State Examination. ${ }^{12}{ }^{13}$ Hitherto, passivity symptoms have received little attention in their own right, making direct comparisons with previous reports difficult. It therefore remains unclear at present whether disturbances of volition might truly be more prominent among post surgical patients than among other epileptic patients having schizophrenia-like psychoses, or among those with an idiopathic schizophrenia.

Case A, who had not had a resective procedure, exhibited depressive delusions congruent with his marked affective symptoms, in addition to some experiences of bodily passivity. Case $\mathrm{F}$ was typical of many patients having the Capgras' syndrome in having his delusions develop subsequent to an affective disturbance with marked derealisation. ${ }^{14}$

Once the psychoses had emerged, they had responded symptomatically at least as well as other psychotic illnesses to appropriate treatment, with the possible exception of case $D$, who, still subject to frequent seizures, showed features of the "alternate psychosis" of epilepsy. ${ }^{15}$ In this, episodes of epileptic 
dyscontrol and florid psychosis appear to substitute for one another, and it is inherently difficult to treat. The social impoverishment found in case $\mathrm{C}$ appeared to be related to an apparent intellectual deterioration that was unlikely to have been a consequence of his psychotic illness.

The appearance of symptoms between the ages of 20 and 31 , as in cases A-E, would be typical of psychotic illnesses unrelated to known cerebral disorder. The Capgras syndrome of case $F$ occurring as it did in the fifth decade, is also typical of that disorder. ${ }^{9}$ While the rapid succession of operation and psychosis in cases $A$ and $F$ suggest that surgery may have been instrumental in the onset of symptoms, the fact that intervals range between one and 9 years for the cases of schizophrenia-like psychosis (cases B-E) suggest that any connection that exists is unlikely to have been a simple one. It remains possible that the surgical operation may have been responsible for the appearance of subsequent psychiatric symptoms in some way.

Although the patients' histories lacked a history of pre-operative psychotic illness, they did contain other features of psychiatric significance. In two cases (C and D) there had been some symptomatic overlap between preoperative phenomena that were apparently ictal (hallucinations and dysphasia) and subsequent psychotic symptoms. In addition, case F's apparently ictal rages were replaced postoperatively by aggressive behaviour in the presence of psychotic symptoms. Only one of the patients (case D) had an unequivocal family history of psychotic illness (possibly of organic origin), but all five others had evidence of emotional disturbance dating back to infancy. Thus cases A, B, C and F had been parentally rejected or abused before onset of their epilepsy, with emotional or behavioural problems being noted in childhood. Case E, whose epilepsy began earliest of all, had been subject to inappropriate emotional attention from his single parent into adult life, and also exhibited life-long behavioural difficulties. The relevance of the family and personal history of temporal lobectomy patients to their predisposition to psychotic illnesses has already been commented upon by Jensen and Larsen in a study notable for the detail of its pre- and postoperative psychiatric assessments. ${ }^{16}$ Of 74 patients undergoing temporal lobectomy, 20 developed a psychotic illness (11 pre-operation; nine post-operation). This proportion was very high, but was linked by those authors to findings that only six patients among the 74 had been psychiatrically "normal" before operation, ${ }^{17}$ and the presence of a family history of "major psychiatric disorders" among near relatives for $65 \%$ of the 20 cases of psychosis.

In our case of Capgras' syndrome (case F), organic factors are hard to ignore. Not only were his symptoms comparable to other Capgras cases, but the neuropathology was similar to that found in other cases that have been associated with cerebral changes. When the mental changes have occurred in the presence of lateralised lesions, these appear to have been exclusively in the non-dominant hemisphere: ${ }^{18}$ our patient had had damage to the non-dominant hemisphere caused by an abscess many years before his right-sided lobectomy.

All our cases indicate a link between the side of operation and the occurrence of postoperative symptoms. This would be a different form of evidence to implicate the operations in the pathogenesis of psychotic symptoms. To our knowledge this report is the only one that reviews consecutive psychiatric referrals, and it is remarkable that surgery was performed to the right hemisphere in all of these cases. Dominance was unequivocally left sided in five cases, but mixed in the sixth (case D). The two largest series of such operations which include data for laterality in published reports, from the Maudsley ${ }^{10}$ and the Rigshospitalet, ${ }^{16}$ account for 323 operations, of which 160 were right sided. Our patients were from three different surgical centres, but there is no reason to suppose that they were drawn from a population biased towards patients having right sided temporal foci.

The occurrence of schizophrenic psychoses following the onset of epilepsy has itself been firmly associated with left-sided foci. Perez and Trimble ${ }^{19}$ collected 139 cases of schizophreniform psychosis from different series in which epilepsy could be localised, and discovered a left sided focus in $112(88 \%)$. The apparent predominance of psychosis among patients having proven right sided lesions is worthy of comment, and bears on the observation already made that the symptoms of these patients seemed atypical of epileptic psychoses in general. Taylor has also noted that among people with epilepsy, females are disproportionately more likely to develop psychoses (50\% of cases occurring in females, despite the greater prevalence of epilepsy in males), ${ }^{20}$ and it was among female patients that the association he discovered between the presence of left handedness and the development of schizophrenia-like psychoses following temporal lobe epilepsy was strongest. ${ }^{21}$ Our patients were all males. Only one (case D) would have been classified as left handed by Taylor's criteria, indicating that different pre-disposing factors for psychosis are likely among our patients.

There are several reports in which right sided temporal surgery has been reported to provoke a higher incidence of subsequent new psychosis than surgery to the left temporal lobe. ${ }^{22}$ From the reports where data for laterality of operation are also available, ${ }^{1023-25}$ new psychoses have followed right sided temporal lobe surgery nearly three times more frequently than left sided temporal lobe surgery. Interestingly, they are complemented by two recent reports involving a total of six cases in which pre-existing schizophrenic symptoms disappeared following surgery. All six had received lobectomies on the left side..$^{25} 26$

Even if the relationship between psychiatric symptomatology and surgical intervention in these patients is not clearcut, the possibility that the trend for outcome to reflect lateralisation could be confirmed is of considerable 
clinical and theoretical significance. For instance, there is a growing literature implicating compromised right-hemisphere function among cases of idiopathic schizophrenic psychoses ${ }^{27}$ which might predict operation on this side to be more deleterious. However, the specific association of epileptic psychoses and left sided foci would require demonstration that a relative right sided hypofunction was induced by left sided epileptic discharge to be consistent with this.

Closer attention should be paid to the psychiatric assessment and follow up of temporal lobectomy patients, so that the impact of surgery on psychiatric symptoms can be researched further.

We are grateful to Drs $T \mathrm{~J}$ Crow and $\mathrm{J}$ R Engel for making records available, and to the Raymond Way Fund for financial support.

1 Bailey P, Green JR, Amador L, Gildes F. Treatment of psychomotor states by anterior temporal lobectomy. Res psychomotor states by anterior temporal lo
Publ Assoc nerv Ment Dis 1953;31:431-6.

2 Falconer MA. The surgical treatment of temporal lobe epilepsy. In: Herrington RN, ed. Current problems in neuropsychiatry. Ashford: Headley Brothers, 1969:

3 Jensen I. Temporal lobe surgery around the world: results, complications, mortality. Acta Neurol Scand 1975; 52:354-73.

4 Milner B. Psychological aspects of focal epilepsy and its management. Adv Neurol 1975;8:299-314.

5 Jensen I. Temporal lobe epilepsy: social conditions and rehabilitation after surgery. Acta Neurol Scand 1976;54:22-44

6 Fenwick PBC. Postscript: what should be included in a standard psychiatric assessment? In: Engel J, ed. Surgical treatment of the epilepsies. New York: Raven Press, treatment of

7 Wing JK, Cooper JE, Sartorius N. The measurement and classitication of psychiatric symptoms. Cambridge: CUP, 1974.

8 Engel JR. Outcome with respect to seizures. In: Engel J, ed. Surgical treatment of the epilepsies. New York: Raven Press, 1987:553-71

9 Christodoulou GN. The syndrome of Capgras. $\mathrm{Br} J$ Psychiatry 1977;130:556-64.

10 Bruton CJ. The neuropathology of temporal lobe epilepsy. Oxford: OUP, 1988:83.

11 Slater E, Beard AW, Glithero E. The schizophrenia-like psychoses of epilepsy. Br J Psychiatry 1963;109:95-150.

12 Perez MM, Trimble MR. Epileptic psychosis-diagnostic comparison with process schizophrenia. Br J Psychiatry 1980;137:245-9.

13 Toone BK, Garralda ME, Ron MA. The psychoses of epilepsy and the functional psychoses: a clinical and phenomenological comparison. Br J Psychiatry 1982; 141:256-61.

14 Trethowan W, Enoch MD. Uncommon psychiatric syndromes Bristol: Wright, 1981.

15 Wolf $P$. Forced normalisation. In: Trimble MR, Bolwig T, eds. Aspects of epilepsy and psychiatry Chichester: Wiley, 1886:101-15.

16 Jensen I, Larsen JK. Psychoses in drug-resistant temporal lobe epilepsy. J Neurol Neurosurg Psychiatry 1979;42: 948-54.

17 Jensen I, Larsen JK. Mental aspects of temporal lobe epilepsy. J Neurol Neurosurg Psychiatry 1979;42:256-65.

18 Cumm anatomical correlations, and review. prenomenology, 1985;146:184-97.

19 Perez M, Trimble MR. Epileptic psychosis: an evaluation of PSE profiles. Br J Psychiatry 1985;146:155-64

20 Taylor DC. Ontogenesis of chronic epileptic psychoses: a reanalysis. Psychol Med 1971;1:247-53.

21 Taylor DC. Factors influencing the occurrence of schizophrenia-like psychosis in patients with temporal lobe

22 Trimble MR. Post-operative psychosis. In: Trimble MR, The Psychoses of Epilepsy. New York: Raven Press, 1991: 91-108.

23 Simmel ML, Counts S. Clinical and psychological results of anterior temporal lobectomy in patients with psychomotor epilepsy. In: Baldwin M, Bailey P, eds. Temporal lobe epilepsy. Springfield: Thomas, 1958:530-50.

24 Polkey CE. Effects of anterior temporal lobectomy apart from the relief of seizures. J Roy Soc Med 1983;76:354-8.

25 Stevens JR. Psychiatric consequences of temporal lobectomy for intractable seizures: a 20-30 year follow up of 14 cases. Psychol Med 1990;20:529-45.

26 Roberts GW, Done DJ, Burton C, Crowe TJ. A “mock-up" of schizophrenia: temporal lobe epilepsy and schizoof schizophrenia: temporal lobe epilepsy and schizo-
phrenia-like psychosis. Biol Psychiatry 1990;28:127-43.

27 Cutting JC. The right cerebral hemisphere and psychiatric disorders. Oxford: Oxford University Press, 1990. 